\title{
Efeitos sedativos das associações xilazina/midazolam e medetomidina/midazolan no gato (Felis catus) ${ }^{1}$. Sedative effects of xylazine/midazolam and medetomidine/midazolam in cats.
}

\author{
Heloisa Justen Moreira de Souza²; Firmino Mársico Filho3; Moyses Fonseca Serpa4; \\ Marcia Maria Barros Espíndola ${ }^{5}$ e Carmen Helena de Carvalho Vasconcellos ${ }^{6}$.
}

\section{Resumo}

Vinte gatos, machos, sem raça definida, com 1 a 2 anos de idade, hígidos, foram divididos em dois grupos iguais. Em ambos os grupos utilizou-se como medicação pré-anestésica o sulfato de atropina, na dose de $0,03 \mathrm{mg} / \mathrm{k}$, por via intramuscular, 20 minutos antes da administração da associação dos alfa-2 agonistas e do midazolam. O primeiro grupo $(\mathrm{x} / \mathrm{m})$ foi submetido à administração intramuscular da associação xilazina/ midazolam, nas doses de $1 \mathrm{mg} / \mathrm{kg}$ e $0,2 \mathrm{mg} / \mathrm{kg}$ e o segundo grupo $(\mathrm{m} / \mathrm{m})$ à associação medetomidina/ midazolam, nas doses de $0,08 \mathrm{mg} / \mathrm{kg}$, e $0,2 \mathrm{mg} / \mathrm{kg}$, respectivamente. A administração simultânea de alfa2 agonistas e midazolam induziu a uma rápida e profunda depressão do SNC, que foi mais rápida com a combinação medetomidina/midazolam. O efeito sedativo máximo ocorreu entre 20-30 min. com ambas as combinações. A recuperação foi tranqüila sem alterações expressivas.

Palavras-chave: sedação, alfa-2 agonista, midazolam, gatos

\section{Introdução}

Os neurônios adrenérgicos centrais desempenham um papel fundamental na regulação da vigília e da dor, e a sedação e a analgesia têm sido uma das conseqüências mais marcantes da ativação dos receptores adrenérgicos tipo alfa-2 (Virtanen, 1989). Desse modo, o emprego dos agonistas dos receptores adrenérgicos alfa-2 vem ganhando um destaque especial na sedação dos felinos. Os benzodiazepínicos são drogas utilizadas em anestesia, tanto na medicação pré-anestésica como para sedação, indução ou manutenção da anestesia geral (Bedin et al., 1994). Outras ações úteis são a atividade anticonvulsivante e capacidade em produzir relaxamento muscular.
Para satisfazer as necessidades de muitos médicos veterinários, na sedação ou como parte de um protocolo anestesiológico para gatos, foram empregados dois agonistas dos receptores adrenérgicos alfa-2, a xilazina e a medetomidina, com um benzodiazepínico, o midazolam, cujo emprego isolado no felino induz a mudanças comportamentais bizarras segundo llkiw et al. (1991a).

O presente estudo teve por finalidade avaliar e comparar os efeitos sedativos das associações xilazina/ midazolam e medetomidina/midazolam no gato.

\section{Material e Métodos}

Foram utilizados 20 gatos, machos, sem raça definida, na faixa etária de 1 a 2 anos, hígidos, com peso corporal que variou entre 3,5 a $5,0 \mathrm{~kg}$, procedentes do gatil do Instituto Biomédico da Universidade Federal Fluminense, previamente selecionados através de exames clínicos e laboratoriais. Este felinos forma mantidos em jejum alimentar de 12 horas e hídrico de 4 horas.

Os animais foram divididos em dois grupos iguais, denominados grupo I e II. Em ambos os grupos, utilizou-se como medicação pré-anestésica o sulfato de atropina (Sulfato de Atropina, 0,25 $\mathrm{mg} / \mathrm{ml}$ - Apsen), na dose de $0,03 \mathrm{mg} / \mathrm{kg}$, por via im., $20 \mathrm{~min}$. antes da adminsitração da associação dos alfa-2 agonistas e do midazolam, segundo Hall e Clarke (1987).

O primeiro grupo $(x / m)$ foi submetido à administração im. da associação xilazina (Rompum, $20 \mathrm{mg} / \mathrm{ml}$ Bayer) / midazolam (Dormonid, $5 \mathrm{mg} / 5 \mathrm{ml}$-Roche), nas doses de $1 \mathrm{mg} / \mathrm{kg}$, de acordo com Muir III e Swanson (1994) e 0,3 mg/kg, conforme llkiw et al. (1991 b). No segundo grupo $(\mathrm{m} / \mathrm{m})$ empregou-se, por via im., a associação medetomidina (Domitor, $1 \mathrm{mg} / \mathrm{ml}$-Farmos Group Ltd) / midazolam, nas doses de 0,08 mg/kg, de acordo com Vähä/Vahe (1989) e $0,2 \mathrm{mg} / \mathrm{kg}$, respectivamente.

\footnotetext{
1 - Trabalho realizado sob os auspicos da CAPES

2 - Prof. Assistente da Universidade Federal Rural do Rio de Janeiro, Instituto de Veterinária - Depto Medicina e Cirurgia, $23851-970$

3 - Prof. Adjunto da Universidade Federal Fluminense - Faculdade de Veterinária - Depto Patologia e Clínica Veterinária, 24230-0340, Niterói, RJ

4 - Médico Veterinário autônomo - RJ

5 - Acadêmica de Medicina Veterinária da Faculdade de Veterináa da Faculdade de Veterinária da UFF

6 - Aluna do Curso de Pós-Graduação em Clínica Cirúrgica da Faculdade de Veterinária da UFF
} 
Os animais foram observados por $180 \mathrm{~min}$. desde a administração das associações dos sedativos até a recuperação.

O período de latência, bem como o período hábil e a recuperação da sedação e a possibilidade de intubação orotraqueal após a administração das associações $x / m$ e $m / m$, foram registrados através dos seguintes parâmetros subjetivos, adaptados de Verstegen et al. (1989):

período de latência (PL): desde a administração das drogas até o momento em que o animal não é capaz de se levantar;

período de indução $(\mathbf{P I})$ : desde a aplicação das drogas até o momento em que for possivel ou não se efetuar a intubação endotraqueal. A intubação endotraqueal foi precedida da dessensibilização da laringe pela utilização do aerossol de lidocaina 10\% (Xylocaina spray $10 \%$-Merrel Lepetit), com uma única aspersão.

período de sedação (PS): período hábil de sedação no qual o animal não é capaz de se levantar;

período de recuperação parcial (PR1): desde a administração das drogas até o momento em que 0 animal se coloca em decúbito esternal;

período de recuperação total (PR2): tempo desde a administração das drogas até o momento que há recuperação da atividade motora;

Foram avaliados a perda ou não dos reflexos oculopalpebral, orofaríngeo, auditivo, deglutição e interdigital, segundo Warren (1986), aos 5, 10, 20, 30, 45, 60, 75, 90, 105 e $120 \mathrm{~min}$. após a aplicação de cada associação. Verificou-se a ocorrência ou não de êmese, excitação, apnéia, micção e defecção. O relaxamento muscular mandibular foi classificado como bom (sem resistência à abertura da boca), moderado (com pequena resistência) e fraco (resistência à aberturá da boca).

Os dados referentes, PL, PI, PS, PR1 e PR2, receberam tratamento estatístico, possibilitando a comparação das médias à nível de $5 \%$, através do teste " $t$ " de Student e de Wilcoxon.

\section{Resultados}

A sedação foi gradual com a administração das duas associações. Inicialmente, os animais apresentaram andar cambaleante e, logo em seguida, sentaram e assumiram a posição de decúbio lateral. Houve diferença significativa $(p<0,05)$ entre as médias obtidas para $\mathrm{PL}$ com as associações $\mathrm{x} / \mathrm{m}$ e $\mathrm{m} / \mathrm{m}$, que foram $5,9 \pm 1,9$ e $3,7 \pm 1,7$ min., respectivamente.

Com a administração da associação $\mathrm{x} / \mathrm{m}$, a duração média da sedação (PS) foi de $85,3 \pm 32,9$ min. e com $\mathrm{m} / \mathrm{m} 91,5 \pm 21,0 \mathrm{~min}$.

As respostas aos estímulos auditivos foram ausentes em $100 \%$ dos gatos que receberam ambas as associações aos $20 \mathrm{~min}$. O reflexo retornou em todos os animais aos $75 \mathrm{~min}$. após a administração da associação $\mathrm{x} / \mathrm{m}$ e em $50 \%$ com a associação $\mathrm{m} / \mathrm{m}$ (Figura 1).

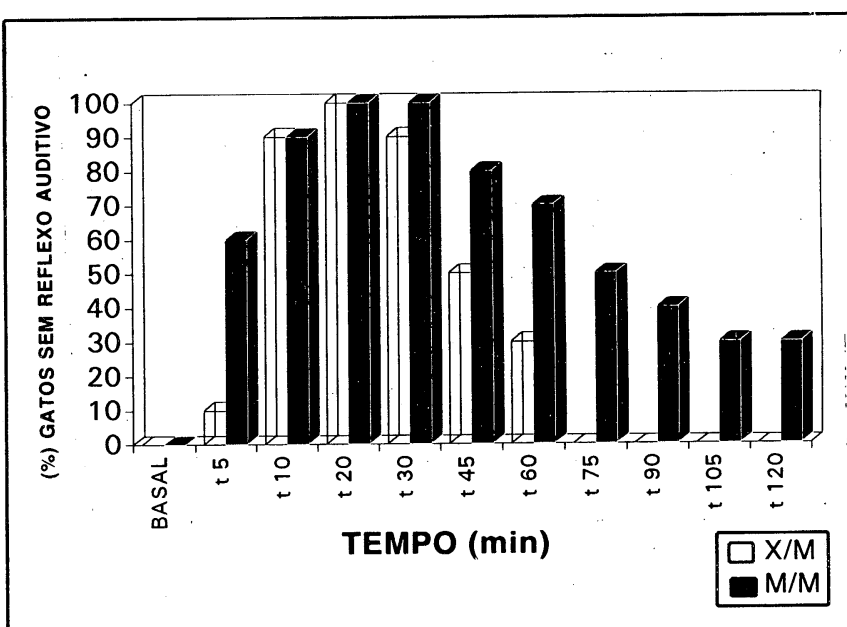

Percentagem de gatos que não apresentou resposta aos estímulos auditivos após a administração de: xilazina/ midazolam e medetomidinina/midazolam.

As pálpebras permaneceram abertas e os globos oculares centralizados durante todo o período de observação. O reflexo oculopalpebral esteve ausente em $80 \%$ dos gatos aos 20 min., e presentes em todos os animais, a partir dos $90 \mathrm{~min}$.

O reflexo de deglutição foi abolido em $60 \%$ dos gatos que receberam $\mathrm{x} / \mathrm{m}$ e em $80 \%$ após o emprego de $\mathrm{m} / \mathrm{m}$ aos 20-30 min., normalizando-se, em ambos os grupos, após $60 \mathrm{~min}$. (Figura 2).

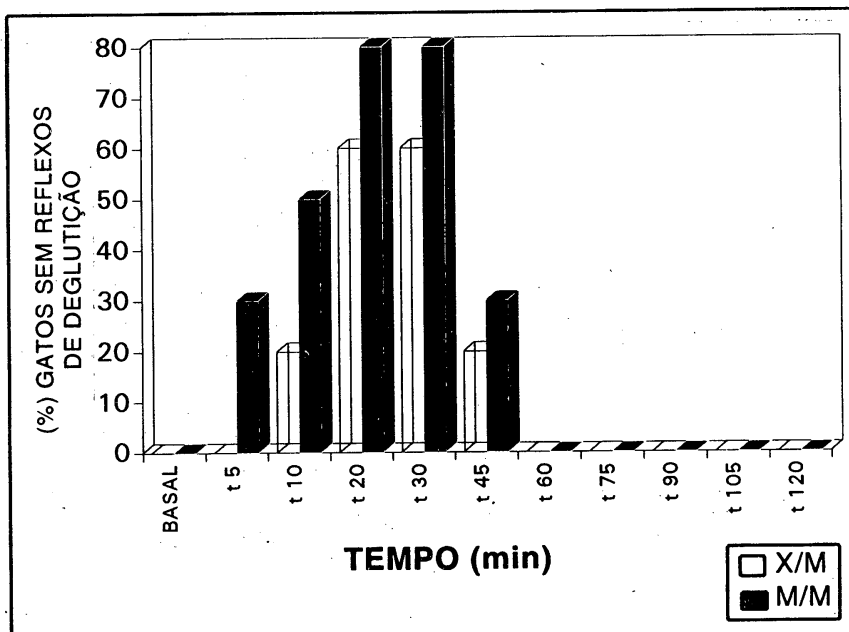

Percentagem de gatos que não apresentou reflexo de deglutição, após a administração de: xilazina/ midazolam e medetomidina/midazolam.

O relaxamento mandibular foi classificado como bom em $60 \%$ dos animais aos 20-30 min.; moderado, 
em $50 \%$ dos animais aos $10 \mathrm{~min}$. e ruim aos $120 \mathrm{~min}$. após a administração da $\mathrm{x} / \mathrm{m}$ (Figura 3 ).

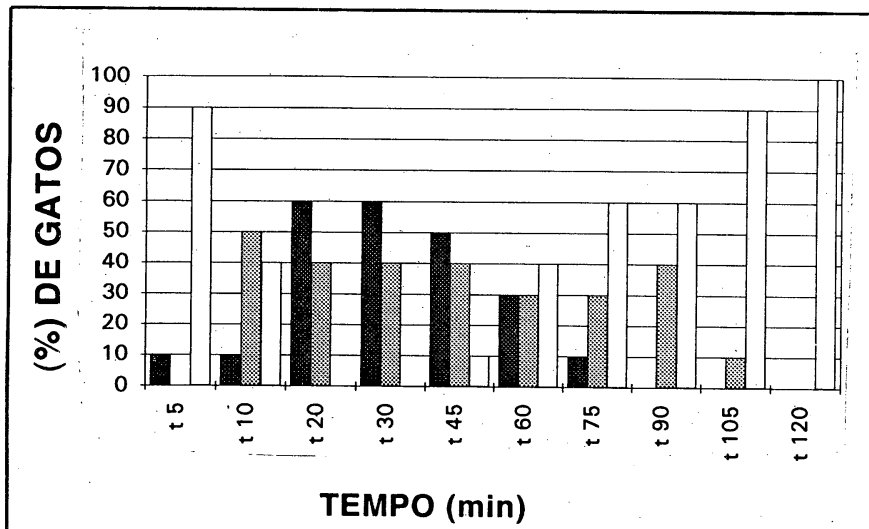

(min)

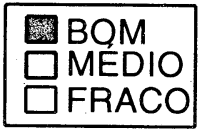

Relaxamento do tono mandibular, após a administraçào de xilazina/midazolam.

Com a associação $\mathrm{m} / \mathrm{m}$, o relaxamento mandibular foi considerado bom em $90 \%$ dos animais aos $30 \mathrm{~min}$. e em $80 \%$ aos $20-45$ min.( Figura 4 ).

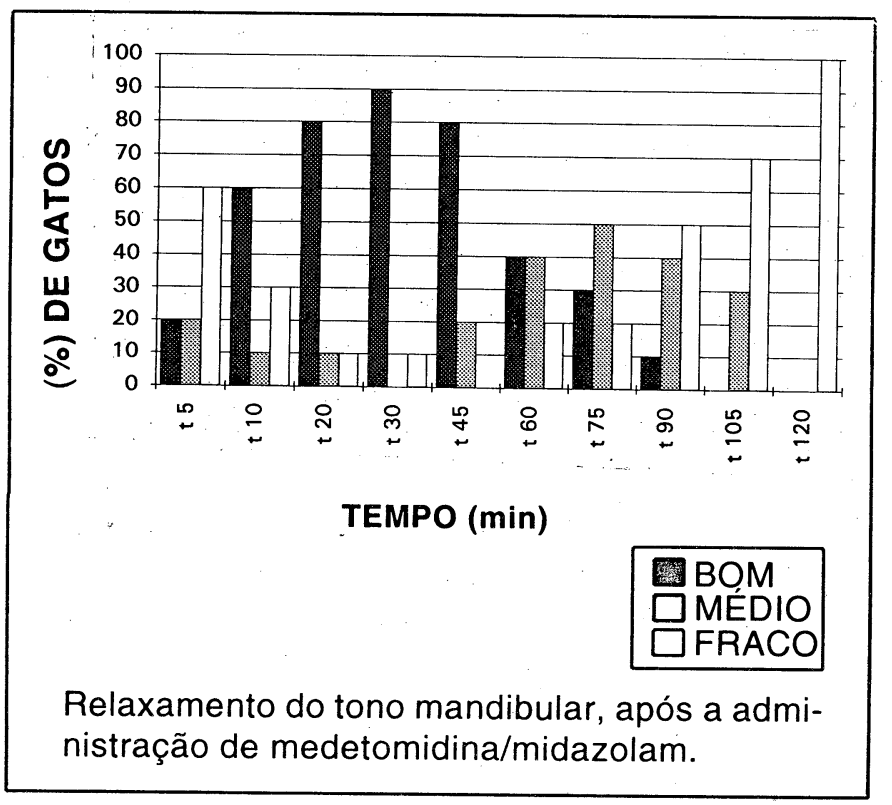

A intubação orotraqueal foi possível em $60 \%$ dos animais com a associação $x / m$, sendo efetuada em média aos $25,5 \pm 6,8 \mathrm{~min}$. e com a associação $\mathrm{m} / \mathrm{m}$ aos $16,1 \pm 6,1 \mathrm{~min}$. em $80 \%$ dos gatos. Houve diferença significativa $(p<0,05)$ entre as médias do $\mathbf{P I}$.

Ao teste de pinçamento dos espaços cutâneos interdigitais dos membros posteriores, a resposta reflexa encontrou-se bastante diminuída em cerca de $90 \%$ dos gatos com a associação $\mathrm{m} / \mathrm{m}$ e em 70 e $80 \%$ dos animais com a associação $x / m$, no intervalo de tempo de 20-30 min. (Figura 5).

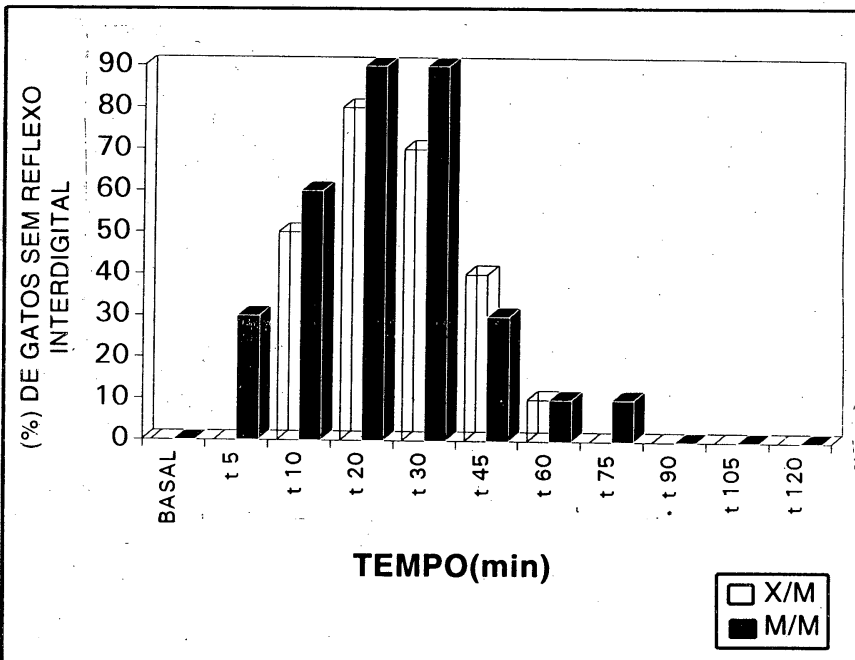

Percentagem de gatos que não apresentou reflexo interdigital, após a administração de: xilazina/. midazolam e medetomidina/midazolam.

A recuperação ocorreu de forma satisfatória. Os gatos assumiram a posição de decúbito esternal (PR1) com a associações $\mathrm{x} / \mathrm{m}$ e $\mathrm{m} / \mathrm{m}$ aos $86 \pm 35,9$ e 104,7 \pm 25,5 min., respectivamente. O PR2 ocorreu com a associação $\mathrm{x} / \mathrm{m}$ aos $125,1 \pm 23,4 \mathrm{~min}$ e $\mathrm{com} \mathrm{m} / \mathrm{m}$ aos $132,5 \pm 24,2 \mathrm{~min}$. Os animais permaneceram ligeiramente sonolentos, mesmo após a recuperação da atividade motora com ambas as associações (Tabela 1).

A êmese foi observada após a administração de $\mathrm{x} /$ $\mathrm{m}$ em $60 \%$ dos gatos aos 5-10 min., enquanto que, com a administração da associação $\mathrm{m} / \mathrm{m}$ ocorreu aos 3-5 min em 50\%. Após o período de recuperação, alguns animais, de ambos os grupos, apresentaram vômitos.

\section{Discussão e Conclusões}

O uso isolado do midazolam em gatos induziu a mudanças comportamentais bizarras, levando a uma difícil aproximação e manipulação, limitando o seu emprego como medicação pré-anestésica (llkiw et al., 1991a). Por este motivo, neste trabalho optou-se pelo emprego das associações das drogas alfa-2 agonistas com o midazolam, concomitantemente e pela via intramuscular. Os gatos não apresentaram reações disfóricas após a aplicação das combinações, como observado por Tranquilli et al. (1990) que empregaram a xilazina em cães alguns minutos antes do midazolam.

Ambas as combinações induziram a uma profunda sedação. Os animais assumiram a posição de decúbito, após a administração de $\mathrm{m} / \mathrm{m}$, num tempo médio significativamente inferior à associação $\mathrm{x} / \mathrm{m}$ e ao uso iso- 
lado da medetomidina em gatos (Short, 1992) e cães (Clarke \& England, 1989). A dose e a via de administração interferem de maneira significativa no tempo que os animais assumem a posição de decúbito (Stenberg et al., 1987; Vähä-Vahe, 1989; Tranquilli et al., 1990). Nesta pesquisa utilizando as menores doses recomendadas de cada fármaco, foi verificado que os períodos de latência de cada combinação foram curtos, sem efeitos colaterais maiores.

O efeito máximo da sedação ocorreu entre o intervalo de 20-30 min., sendo ligeiramente maior com a combinação $\mathrm{m} / \mathrm{m}$. Com a administração da combinação $x / m$, a depressão dos reflexos não foi tão duradoura. Vähä-Vahe (1989), utilizou as doses de medetomidina em gatos no intervalo de 0,05-0,15 mg/ $\mathrm{kg}$ por via im. e $85 \%$ dos animais encontraram-se em estado de sedação profunda com duração de 60 a 120 min. e quando foram empregadas doses mais elevadas, o período de sedação excedeu os 120 min., não reagiram a ruídos externos, à semelhança do que foi relatado por Vähä-Vahe (1990), quando utilizou 0,1 mg/ $\mathrm{kg}$ de medetomidina. Newkirk \& Miles (1974) citaram que o período de sedação total com a administração da xilazina é de 60 a 120 min., podendo chegar a 180 min., sendo então dose-dependente. Alguns gatos responderam aos estímulos auditivos externos. 0 midazolam, administrado nas doses 2,0 e $5,0 \mathrm{mg} / \mathrm{kg}$ por via im. em gatos, produziu mudanças comportamentais psicomotoras, ficando evidentes por um período de tempo de 120 min. (llkiw et al., 1991a). Com base nas observações clínicas dos efeitos das drogas supracitadas, infere-se que a duração da sedação das associaçõés $\mathrm{x} / \mathrm{m}$ e $\mathrm{m} / \mathrm{m}$ não diferem substancialmente das drogas quando utilizadas separadamente, por outro lado, a ausência ou diminuição de resposta aos estímulos auditivos e demais reflexos, indicam a ocorrência de um efeito aditivo entre as drogas administradas nos dois grupos aumentando a intensidade do efeito sedativo e não a sua duração.

A intubação orotraqueal foi possível e sendo efetuada num período de tempo mais curto após a administração de $\mathrm{m} / \mathrm{m}$. Por outro lado, Hellyer et al. (1989) obtiveram tempos menores na intubação endotraqueal com a associação midazolam/quetamina (2,73 min.) e diazepam/quetamina (4,07 $\mathrm{min}$.) em cães, enquanto que, llkiw et al (1991 b) utilizaram as doses de midazołam $(0,5$ e $5,0 \mathrm{mg} / \mathrm{kg})$ e uma dose fixa de quetamina $(3,0 \mathrm{~kg} / \mathrm{mg})$ por via iv. em gatos e relataram que $83 \%$ dos animais não apresentaram reflexo de deglutição aos 6 min. Clarke e England (1989) quando utilizaram a medetomidina em cães conseguiram um relaxamento do tono muscular mandibular suficienté para examinar a região faringeana, mas mesmo nos cães profundamente sedados não foi possível a intubação endotraqueal. O mesmo ocorreu quando. Castro et al. (1988) administraram o midazolam em cães na dose de $2,0 \mathrm{mg} / \mathrm{kg}$ por via iv., decorridos 15 min. da injeção de levomepromazina. No presente estudo, observou-se que o reflexo laringotraqueal foi ligeiramente diminuído, mas não abolido, o que só ocorreu, após o uso do anestésico local, à semelhança do relatado por Short (1994), quando ao emprego de quetamina e tiopental em gatos. O PI médio para a intubação orotraqueal após a administração das associações $\mathrm{x} / \mathrm{m}$ e $\mathrm{m} / \mathrm{m}$ foi maior do que com o emprego do tiopental e da quetamina (Clarke \& England, 1989), porém, a facilidade de aplicação destas combinações por via im. e a aparente boa aceitação por parte dos felinos, faz com que possam ser utilizadas em alguns animais, não somente para obtenção de uma sedação profunda, mas também, como parte de um protocolo anestésico.

A êmese observada nos animais, também citada por Klide et al. (1975) quanto ao uso da xilazina e Vainio et al. (1989) com a medetomidina, segundo Hikasa et al. (1989), decorreu da estimulação dos receptores alfa-2, situados provavelmente na área postrema e não por outros receptores. O midazolam não possui ação emética (Reves et al., 1985; lamele, 1990). Nesta pesquisa, alguns gatos apresentaram êmese após o período de recuperação, com ambas as associações, o que foi relatado por Vainio et al. (1989), quanto ao uso isolado da medetomidina, o que nos faz supor, que o efeito emético dos alfa-2 pode perdurar por um tempo maior do que o da sedação.

O uso das associações $\mathrm{x} / \mathrm{m}$ e $\mathrm{m} / \mathrm{m}$ proporcionou uma recuperação tranqüila e sem tremores, o que está de acordo com Tranquilli et al. (1990), e em contraste com a associação de quetamina / midazolam (llkiw et al., 1991b) e quetamina / xilazina (Duke et al., 1988). Os agonistas dos receptores adrenérgicos alfa-2 desempenharam um papel importante na obtenção de uma recuperação gradual e do estado de sonolência que os animais assumiram, o que coincide com os relatos de Clarke e England (1989). Entretanto, o uso da midazolam pode potencializar a ação dos alfa-2 agonistas, o que é corroborado por Tranquilli et al. (1990).

Os resultados obtidos com as associações $\mathrm{x} / \mathrm{m}$ e $\mathrm{m} / \mathrm{m}$ em gatos, nas doses e técnicas utilizadas, permitiram apresentar as seguintes conclusões:

- a combinação $\mathrm{m} / \mathrm{m}$ promoveu uma profunda sedação com um período de latência mais curto do que com a combinação $\mathrm{x} / \mathrm{m}$;

- a intensidade da sedação foi maior com a associação $\mathrm{m} / \mathrm{m}$;

- o efeito sedativo máximo com ambas as associações ocorreu entre o intervalo de tempo de 20-30 minutos;

- a depressão no sistema nervoso central não foi suficiente para que houvesse a perda do reflexo laringotraqueal. 
Tabela 1 - Comparação entre as médida dos Períodos de Latência, Indução, Sedação, Recuperação parcial e Recuperação total após a administração das associações xilazina/midazolam e medetomidina/midazolam através do teste "t" de Student e Wilcoxon.

\begin{tabular}{|c|c|c|c|c|c|c|c|}
\hline \multirow{2}{*}{\multicolumn{2}{|c|}{$\frac{\text { DROGA }}{\text { PARÂMETRO }}$}} & \multirow{2}{*}{\multicolumn{2}{|c|}{$\frac{X / M \quad M / M}{\text { MÉDIA ( } \pm \text { devio padrão) } \min .}$}} & \multicolumn{4}{|c|}{ Comparação entre os dois tratamentos } \\
\hline & & & & \multicolumn{2}{|c|}{$\begin{array}{l}\text { TESTE " } \mathrm{t} \text { " } \\
\text { Estatística t Prob > /t/ }\end{array}$} & \multicolumn{2}{|c|}{$\begin{array}{l}\text { TESTE WILCOXON }\left(^{*}\right) \\
\text { Estatística Z Prob }>\text { IZI }\end{array}$} \\
\hline PL & \multicolumn{2}{|c|}{$5,9 \pm 1,9$} & $3,7 \pm 1,7+$ & $-2,6723$ & 0,0155 & 2,2634 & 0,0236 \\
\hline P. I. & \multicolumn{2}{|c|}{$25,5 \pm 6,8$} & $16,1 \pm 6,1+$ & $-2,7045$ & 0,0191 & 2,2667 & 0,0234 \\
\hline P. S. & \multicolumn{2}{|c|}{$85,3 \pm 32,3$} & $91,5 \pm 21,0$ & 0,5012 & 0,6223 & $-0,4915$ & 0,6230 \\
\hline PR1 & \multicolumn{2}{|c|}{$87 \pm 35,9$} & $104,7 \pm 25,5$ & 1,2708 & 0,2200 & $-1,5508$ & 0,1209 \\
\hline PR2 & \multicolumn{2}{|c|}{$12,1 \pm 23,4$} & $132,5 \pm 24,2$ & 0,6938 & 0,4966 & $-0,8713$ & 0,3836 \\
\hline
\end{tabular}

(*) Aproximação normal

$(t)$ Diferença significante entre

as médias obtidas $(p<0,05)$

\section{Abstract \\ Sedative Effects of xylazine/midazolam and medetomidine/midazolam in cats.}

Twenty healthy tom cats, from 1 to 2 years of age and weighting between 3,5 and $5 \mathrm{~kg}$ were used in the study. The animals were divided into two groups and were premedicated with $0,03 \mathrm{mg} / \mathrm{kg}$ of atropine sulfate i.m. $20 \mathrm{~min}$ prior to the administration of $1 \mathrm{mg} / \mathrm{kg}$

\section{Referências Bibliográficas}

BEDIN, A., SILVA Jr, I.J., VIDEIRA, R.L.R, CASTO, R.A.C., TURAZZI, J.C., RICHTER. L..R. A aminofilina antagoniza a ação do midazolam. Rev. Bras. Anestesiol., v. 44, n. 5, p. 309-313, 1994.

CASTRO, G.B., MASSONE, F., LUNA, S.P.L., AGUIAR, A.J.A.. Efeitos sobre o equilíbrio ácido básico e gases sangüíneos após o uso de midazoolam em cães. Ars Vet, v. 4, n. 1, p. 9-14, 1988.

CLARKE, K.W., ENGLAND, G.C.W. Medetomidine, a new sedative-analgesic for use in the dog and its reversal with atipamezole. Journal of Small Animal Practice, v. 30, n. 6, p. 343-348, 1989.

DUKE T., HALE, G.J.., JONES, R.S. Clinical Observations on the Simultaneous Administrion of Xylazine and Ketamine for Anesthesia in the Cat. Companion Animal Practice, v. 2, n. 8, p. 3-6, 1988.

ENGLAND, G.C.W., CLARKE, K.W. The uso of Medetomidine/Fentanil Combinations in dogs. Medetomidine (Domitor Symposium, Turku, Finland, June 14-15, 1988. Acta Vet Scand., v. 85, p. 179186, 1989. Suplemento. xykazine and $0,2 \mathrm{mg} / \mathrm{kg}$ midazolam (group $\mathrm{m} / \mathrm{m}-\mathrm{n}=$ 10) i.m.. The combination of alfa2 agonists and midazolam induced a rapid CNS depression. The onset of sedation was earlier with $\mathrm{m} / \mathrm{m}$ than $\mathrm{x} / \mathrm{m}$. The maximal sedative effect occurred at 20 to $30 \mathrm{~min}$ with the two combinations and recovery was uneventful.

Key-words: sedative, alpha-2 agonist, midazolam, cats

HALL, L.W., CLARKE, K.W. Anestesia Veterinária, 8ed. São Paulo: Manole, 1987. 440p. Cap. 16: Anestesia do gato, p. 371-386.

HELLYER, P.W., HUBBELL, J.A.E., FREEMAN, L.C., ABRAHAMSEN,M E.J. Induction of anesthesia with diazepam-ketamine versus midazolam-quetamine in Greyhounds. Veterinary Surgery, v. 18, n. 2, p. 167, 1989.

HIKASA, Y., TAKASE, K, OGASAWARA, S. Evidence for the involviment of alpha2-adrenoceptors in emetic action of xylazine in cats. Am. J. Vet. Res, v. 50, n. 8, p. $1348-1351,1989$.

IAMELE, G.E. Las Drogas Traquilizantes en Medicina Veterinaria, Buenos Aires: Inter-Vet, 1990. 199p.

ILKIW J.E, SUTTER, C., MCNEAL, D., STEFFEY, E.P. Behavioral effects of Midazolam following intravenous and intramuscular administration in health awake cats. Veterinay Surgery. v. 20, n. 2, p.. 157, 1991a. Abstract.

ILKIW, J.E., SUTTER, C., MCNEAL, D., STEFFEY, E.P. Effect of intravenous administration of variable-dose Midazolam following fixed-dose ketamine in healthy awake cats. Veterinay Surgery, v. 20, n. 2, p. 157, 1991b. Abstract. 
KLIDE, A.M., CALDERWOOD, H.W., SOMA L.R. Cardiopulmonary effects of xylazine in dogs. Am. J. Vet. Res. v. 36, n. 7, p. 931-935, 1975.

MUIR III, W.W., SWANSON, C.R. Principles and Techniques of anesthesia and Chemical Restraint. IN: Sherding, R. G. The Cat :Disease and Clinical Management, 2ed. Nova York: Churchill Livingstone, 1994. 2 v., 2046 p. v. 1. Cap. 5, p. 91132.

NEWKIRK, H.L., MILES, D.G. Xylazina as a sedativeanalgesic for dogs and cast. Mod. Vet. Pract, v. 55, n. 9, p. 677-680, 1974.

REVES, J.G., FRAGEN, R.J., VINIK, H.R., GREENVLATT, D.J. Midazolan:pharmacology and use. Anesthesiology, v. 62, n. 3, p, 310-324, 1985.

SHORT, C.E. Alpha-2-Agents in Animals: sedation, analgesia and anaesthesia. Santa Barbara: Veterinary Practice Publishing, 1992. 85 p. Cap. 4: Alpha2-Adrenergic Agonists/Antagonists in Cats, $p$. 59-70.

SHORT, C.E. Pain and its Control in Small Animalsdiagnostic, medical, or minor Sugery Management. In: The North American Veterinary Conference, 1994, Orlando, Florida. Proceedings... Orlando: ESVA, 1994. P. 35-41.

STENBERG, D., SALVÉN, P. MIETTINEN, M.V.J. Sedative action of the alphs2-agonist medetomidine in cats. J. vet. Pharmacol. Therap. v. 10, n. 4, p. 319-323, 1987.

TRANQUILLI, W.J., GROSS, M.E., THURMON, J.C., BENSON, G.J. Evaluation of three MidazolamXylazine Mixtures. Preliminary Trials in dogs. Veterinay Surgery. v. 19, n. 2, p. 168-172, 1990.

VÄHÄ-VAHE, T. Clinical Evaluation of Medetomidine, a Novel Sedative and Analgesic Drug for Dogs and Cats. Acta Vet. Scand., v. 30, n. 3, p. 267-273, 1989.

VÄHÄ-VAHE, T. Clinical effectiveness of atipamezole as a medetomidine antagonist in cats. Journal of Small Animal Practive, v. 31, n. 4, p. 193-197, 1990.

VAINIO, O., VÄHÄ-VAHE, T., PALMU, L. Sedative and analgesic effects of medetomidine in dogs. J. Vet. Pharmacol. Therap, v. 12, n. 2, p. 225-231, 1989.

VERSTEGEN J., FARGETTON, X., ECTORS, F. Etude comparative de diversas associations anesthésiques chez le chat. Caractérisation d'une nouvelle association: médétomidine/Kétamine. Ann. M';ed. Vét., v. 133, n. 1, p. 45-54, 1989.

VIRTANEN, R. Pharmacological Profiles of Medetomidine and its Antagonist, Atipamezole. Medetomidine (Domitor) Symposium, Turku, Finland, June 14-15, 1988. Acta vet Scand, v. 85, p. 29-37, 1989. Suplemento.

WARREN, R. G. Anestesia dos animais domésticos, Barcelona: Labor, 1986. 328p. 